\title{
Aves del municipio de Santiago de Cali, Valle del Cauca, Colombia
}

\author{
Birds of Santiago de Cali, Valle del Cauca, Colombia \\ Rubén D. Palacio, Viviana Vidal-Astudillo, Giovanni Cárdenas y José Luna-Solarte
}

\section{Resumen}

Se presenta un listado de las especies de aves con registros históricos y actuales en el área de Santiago de Cali, el municipio de mayor importancia en la cordillera Occidental de Colombia, con una superficie total de $561,7 \mathrm{~km}^{2}$ y elevaciones desde 950 hasta 4070 m s.n.m. Mediante una recopilación y revisión de referencias bibliográficas, bases de datos de las colecciones ornitológicas del municipio y la base de datos ciudadana de eBird, se obtuvo un listado de 561 especies, que incluye 487 aves residentes, 72 migratorias neártico-neotropicales y dos especies introducidas que se han establecido. Otras 25 especies tienen registros pero requieren de más evidencias para su inclusión, y existen vacíos de información en el PNN Farallones de Cali, la ribera del río Cauca y humedales. En el listado se registran siete especies endémicas de Colombia, 52 casi-endémicas, 22 amenazadas a nivel nacional y 26 a nivel global. A nivel regional en el Valle del Cauca 86 se encuentran amenazadas y seis están presuntamente extintas. Nuestros resultados ubican al municipio de Santiago de Cali como un área de alto valor ornitológico en Colombia y se identifican prioridades de investigación y oportunidades de conservación para garantizar la permanencia de la avifauna municipal y regional.

Palabras clave. Avifauna regional. Conservación. Listado taxonómico. Ornitología urbana. Suramérica.

\begin{abstract}
A list of bird species with historic and current records in Santiago de Cali is presented. Santiago de Cali is the main city in the Western Andes mountain range of Colombia, with a total area of $561.7 \mathrm{~km}^{2}$ and an altitudinal range from 950 to 4,070 $\mathrm{m}$ a.s.l. A list of 561 species was obtained based on a revision and compilation of bibliographic references, databases of regional ornithological collections, and the eBird citizen science platform. These species include 487 breeding residents, 72 nearctic-neotropical migrants, and two introduced species. Records for another 25 species were found, but need further evidence in order to be included. There are information gaps in the National Natural Park Farallones de Cali, the banks of the Cauca River and wetlands in the area. The list presented here includes seven Colombian endemics, 52 near-endemics, 22 nationally threatened birds, and 26 species globally threatened. At the regional level in the state of Valle del Cauca 86 birds are threatened and six are potentially extinct. These results place Santiago de Cali as an area of high ornithological importance, as well as it identifies research and conservation priorities to guarantee the long-term presence of avifauna in the region.
\end{abstract}

Key words. Conservation. Regional avifauna. South America. Taxonomic list. Urban ornithology. 


\section{Introducción}

Los listados de especies aportan información de línea base para el conocimiento de la biodiversidad, $\mathrm{y}$ en años recientes se han usado para estudios de ornitología urbana en varios países de Latinoamérica, aunque en Colombia el conocimiento de la avifauna presente en las ciudades todavía es limitado (OrtegaÁlvarez y MacGregor-Fors 2011, Delgado y Correa 2013). Esta situación disminuye la capacidad para la toma de decisiones que promuevan la sostenibilidad ambiental a nivel regional, donde las ciudades son las encargadas de liderar estos procesos (Andrade-Medina y Bermúdez-Cárdenas 2010). En el municipio de Santiago de Cali se han adelantado algunos trabajos recopilatorios en libros e informes técnicos sobre áreas urbanas (Vidal-Astudillo et al. 2008, Dagma 2010), suburbanas (Vidal-Astudillo 2013) y rural (Vidal-Astudillo 2010). Sin embargo, estos trabajos no permiten cuantificar adecuadamente la riqueza de aves, debido a que no hacen una revisión de los registros obtenidos en la compilación para determinar su validez. Este documento representa entonces el primer listado revisado sobre las aves del municipio de Santiago de Cali con registros históricos y actuales que abarca toda el área en sus componentes urbano, suburbano y rural.

\section{Material y métodos}

\section{Área de estudio}

El municipio de Santiago de Cali está ubicado sobre el piedemonte de la vertiente oriental de la cordillera Occidental (coordenadas 327'26”N- 76 31'42”O; Figura 1). Cubre una superficie total de $561,7 \mathrm{~km}^{2}$, y abarca un amplio gradiente altitudinal con diferentes ecosistemas desde el bosque seco tropical y madreviejas en el valle geográfico del río Cauca-VGRC a $950 \mathrm{~m}$ s.n.m. hasta bosques altoandinos y páramos llegando a los 4070 m s.n.m. sobre las cumbres de los Farallones de Cali, en límites con el municipio de Buenaventura (Dapm 2016). Debido a esta cercanía con la región del Pacífico, Santiago de Cali contiene elementos bióticos del Chocó biogeográfico que recibe a través de las zonas bajas de la cordillera. Solo el 21,6 \% de la superficie son suelos urbanos y la mayor parte es área rural (Dapm 2016). La precipitación es de 1200 $\mathrm{mm} / \mathrm{año} \mathrm{en} \mathrm{las} \mathrm{áreas} \mathrm{planas} \mathrm{y} \mathrm{alcanza} \mathrm{hasta} 3000 \mathrm{~mm} /$ año en los Farallones de Cali. La temperatura media anual es $24{ }^{\circ} \mathrm{C}$ y en las partes más altas es de $15{ }^{\circ} \mathrm{C}$ (Ciat et al. 2016).

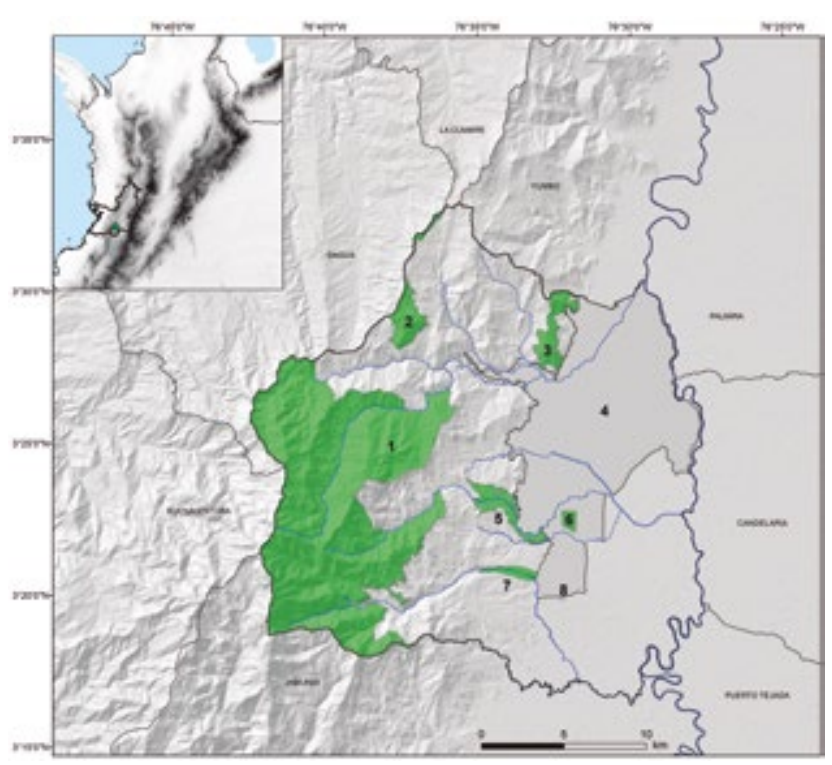

Figura 1. Municipio de Santiago de Cali, Valle del Cauca, Colombia y localidades representativas con trabajo ornitológico. 1- Parque Nacional Natural Farallones de Cali. 2- IBA-Bosque de San Antonio/Km 18. 3- Ecoparque Cerro de las Tres Cruces-Bataclán. 4- Zona Urbana de Cali (Nororiental). 5- Reserva Municipal de Uso sostenible Río Meléndez. 6- Universidad del Valle-Campus Meléndez. 7- Ecoparque Río Pance. 8- Comuna 22 y complejo de humedales (eg. Ecoparque Lago de las Garzas, Lago de Los Cisnes -o de La Babilla-)

Dentro de las áreas de conservación y protección ambiental del municipio, se destacan en la zona rural el Parque Nacional Natural Farallones de Cali, una de las áreas protegidas más biodiversas del país (UAESPNN 2005) y el bosque de San Antonio/Km 18 , el cual es un Área Importante de Aves y Biodiversidad (Important Bird and Biodiversity Area-IBA) (BirdLife International 2017) y uno de los pocos sitios donde se han realizado estudios ornitológicos por más de 100 años (Kattan et al. 2016). En las zonas urbanas y suburbanas sobresalen por su importancia los 10 ecoparques de la ciudad (Dapm 2016), algunos de ellos con ambientes acuáticos como el ecoparque río Pance y 
el ecoparque Lago de Las Garzas, y otros que protegen los cerros tutelares como los ecoparques Cristo Rey en el cerro Los Cristales, el cerro de las Tres CrucesBataclán y el cerro de La Bandera.

\section{Elaboración del listado}

Se recopiló y revisó información sobre las aves registradas en el municipio de Santiago de Cali mediante consulta por referencias bibliográficas, bases de datos de las colecciones ornitológicas de la ciudad y la base de datos de ciencia ciudadana eBird (Sullivan et al. 2009), donde también se encuentran registradas las observaciones de los autores (Anexo 1 DOI: 10.21068/ c2017.v18n02a15a01).

1. Referencias bibliográficas: se hizo una búsqueda exhaustiva y recopilación de registros en artículos científicos, informes técnicos, trabajos de grado, planes de manejo, listados de especies y otras fuentes de literatura gris, así como guías de identificación y libros con reportes de aves del municipio.

2. Colecciones de referencia: se obtuvieron las bases de datos de los registros de especímenes depositados en las dos colecciones ornitológicas más importantes del municipio con el fin de evaluar su representatividad la Colección Ornitológica de la Universidad del Valle (Universidad del Valle 2014) y Colección Zoológica de referencia "IMCN" del Museo de Ciencias Naturales "Federico Carlos Lehmann V." (Riascos-Vallejo 2001).

3. Base de datos ciudadana de eBird: se obtuvo registros para el departamento del Valle del Cauca hasta noviembre de 2016 (eBird 2016) y se filtraron para el municipio de Santiago de Cali con base en las coordenadas límite: 3.27, 3.55 / -76.71, -76.45 (Latitud mínima, máxima / Longitud mínima, máxima).

Como herramienta de compilación de la información, se construyó una base de datos relacional-BDR en Microsoft Access. Esta herramienta se estructuró para el ingreso de los listados de aves de la fuente original de una manera estandarizada, así como para la verificación y actualización de la información de cada una de las especies de aves registradas, lo que resultó muy útil para minimizar errores en el manejo de datos. La información final se exportó a una hoja de cálculo en Excel para su análisis. Para validar el registro de las especies y asegurar el rigor científico de la lista, se tuvieron en cuenta de manera conjunta criterios de inclusión como el soporte de evidencias audiovisuales, número de registros por especie y si la presencia de la especie es congruente con su rango de distribución conocido o se encuentra dentro del área accesible por dispersión (Barve et al. 2011).

\section{Resultados}

\section{Listado de aves del municipio de Santiago de Cali}

Se obtuvo un listado de 561 especies de aves (Anexo 2 DOI: 10.21068/c2017.v18n02a15a01), pertenecientes a 24 órdenes, 63 familias y 346 géneros, lo cual equivale al 30,4\% de las 1847 especies registradas para Colombia de acuerdo al Comité de Clasificación de América del Sur (SAAC, por sus siglas en Inglés) (Remsen et al. 2017). La lista comprende 487 especies Residentes (R), 72 Migratorias Latitudinales (Mla, neárticas-neotropicales) (Naranjo et al. 2012) y dos especies Introducidas (In) que se consideran establecidas (Columba livia y Lonchura malacca). También se identificaron registros de interés de especies poco conocidas y aquellas que requieren extensión del rango de distribución y elevación.

De interés para la conservación, se encontraron siete especies endémicas (E), 52 casi-endémicas (CE), tres especies de interés (EI) y una especie con información insuficiente (II) (Chaparro-Herrera et al. 2013). En cuanto a las categorías de amenazas de extinción, a nivel regional se encuentran 86 especies amenazadas y seis especies presuntamente extintas (SX) (CastilloCrespo y González-Anaya 2007). A nivel nacional hay dos especies En Peligro Crítico (CR), seis En Peligro (EN) y nueve como Vulnerables (VU), cinco Casi Amenazadas (NT), 14 con Preocupación Menor (LC) y tres con Datos Deficientes (DD) (Renjifo et al. 2014, 2016). A nivel global el municipio presenta tres especies En Peligro (EN), nueve especies Vulnerables (VU), 14 especies Casi Amenazadas (NT), 534 especies 
con Preocupación Menor (LC) y una especie con Datos Deficientes (DD) (IUCN 2016).

De la recopilación realizada se excluyeron 69 especies con registros en los listados obtenidos (Anexo 2). De estas, 25 especies se consideraron potenciales para el municipio, y requieren de evidencia tangible para ser incluidas, porque la distribución conocida no respalda el registro o son posibles confusiones en la identificación respecto a otras especies ya corroboradas. Las restantes 44 especies son excluidas porque corresponden a errores de identificación o son introducidas que no se han establecido y en consecuencia no hacen parte de la avifauna del municipio (Anexo 2).

De las 561 especies confirmadas para el municipio de Santiago de Cali, la base de datos eBird (2016) tiene la mayor representatividad de especies con el 89,1\% (500), seguida por las referencia bibliográficas que reportan el 87,5 \% (491), la Colección Ornitológica de la Universidad del Valle reporta el 45,8 \% (257) y la Colección Zoológica de referencia "IMCN" del Museo de Ciencias Naturales "Federico Carlos Lehmann V." reporta el 36,2 \% (203), ambas colecciones tienen una representatividad de 56,9\% (319, compartiendo 140 especies) y los registros aislados o adicionales son el $0,9 \%(5)$.

\section{Discusión}

\section{Riqueza de especies}

Debido a su amplio rango altitudinal y posición geográfica privilegiada, en Santiago de Cali se han registrado el 30,4\% de las aves de Colombia en menos del 0,05\% de su superficie terrestre, y se ubica como el municipio con mayor riqueza de aves para el país de los estudiados actualmente con 561 especies. Le siguen Ibagué (537 spp, Parra-Hernández et al. 2007), Medellín (445 spp, Estrada 2014), Manizales (439 spp, Verhelst et al. 2001, Botero et al. 2005), Popayán (338 spp, Ayerbe-Quiñones et al. 2009) y Pereira (203 spp, Londoño-Betancourth 2011). Bogotá tiene alrededor de 269 especies (Andrade-L y BenítezCastañeda 2002). Se encontró que el municipio de Santiago de Cali tiene una riqueza de especies similar a departamentos de Colombia con inventarios y estudios intensivos en ornitología como el Quindío (560 spp, Arbeláez-Cortés et al. 2011, 2015). Sin embargo, la avifauna del municipio todavía no está completa. En la elaboración del listado se identificaron 25 especies con registros que necesitan ser verificados con más evidencias (potenciales); y mediante adiciones con nuevos inventarios en áreas poco muestreadas, se estima que la avifauna de Santiago de Cali podría superar las 600 especies.

\section{Vacíos de información}

Para el municipio existe un vacío de información hacia las zonas del PNN Farallones de Cali por encima de los $2500 \mathrm{~m}$, en bosques altoandinos y páramos donde se podrían corroborar especies potenciales, obtener registros actualizados de especies que no han sido observadas en los últimos años y nuevas adiciones de especies para el municipio (Figura 2). Por ejemplo, exploraciones recientes en la localidad del Alto del Buey $(3500 \mathrm{~m})$ han permitido encontrar nuevos registros tales como Ramphomicron microrhynchum, Eriocnemis vestita y Colaptes rivolii. Otras especies como Asthenes fuliginosa y Atlapetes pallidinucha tienen registros en el páramo del Duende (Estela et al. 2004), y podrían estar presentes en el municipio. También existe la posibilidad de encontrar Henicorhina negreti y Diglossa gloriosissima, ambas especies endémicas de Colombia y en peligro de extinción, donde esta última se ha sugerido que puede estar en los picos del PNN Farallones de Cali (Renjifo et al. 2014). Intensificar los muestreos en tierras altas permitiría tener una mejor capacidad de análisis ecológicos y evaluar estatus de conservación tanto de las especies como de los ecosistemas que por el momento se encuentra limitada por la falta de registros actualizados y confiables en la zona.

Otras áreas que requieren mayor exploración son las zonas bajas de la cordillera con influencia del Chocó montano donde se podrían encontrar especies como Heliodoxa imperatrix o Pseudocolaptes lawrencii. También se deben intensificar muestreos en la ribera del río Cauca y las madreviejas o humedales existentes en la zona plana, que puede contener nuevos registros de 


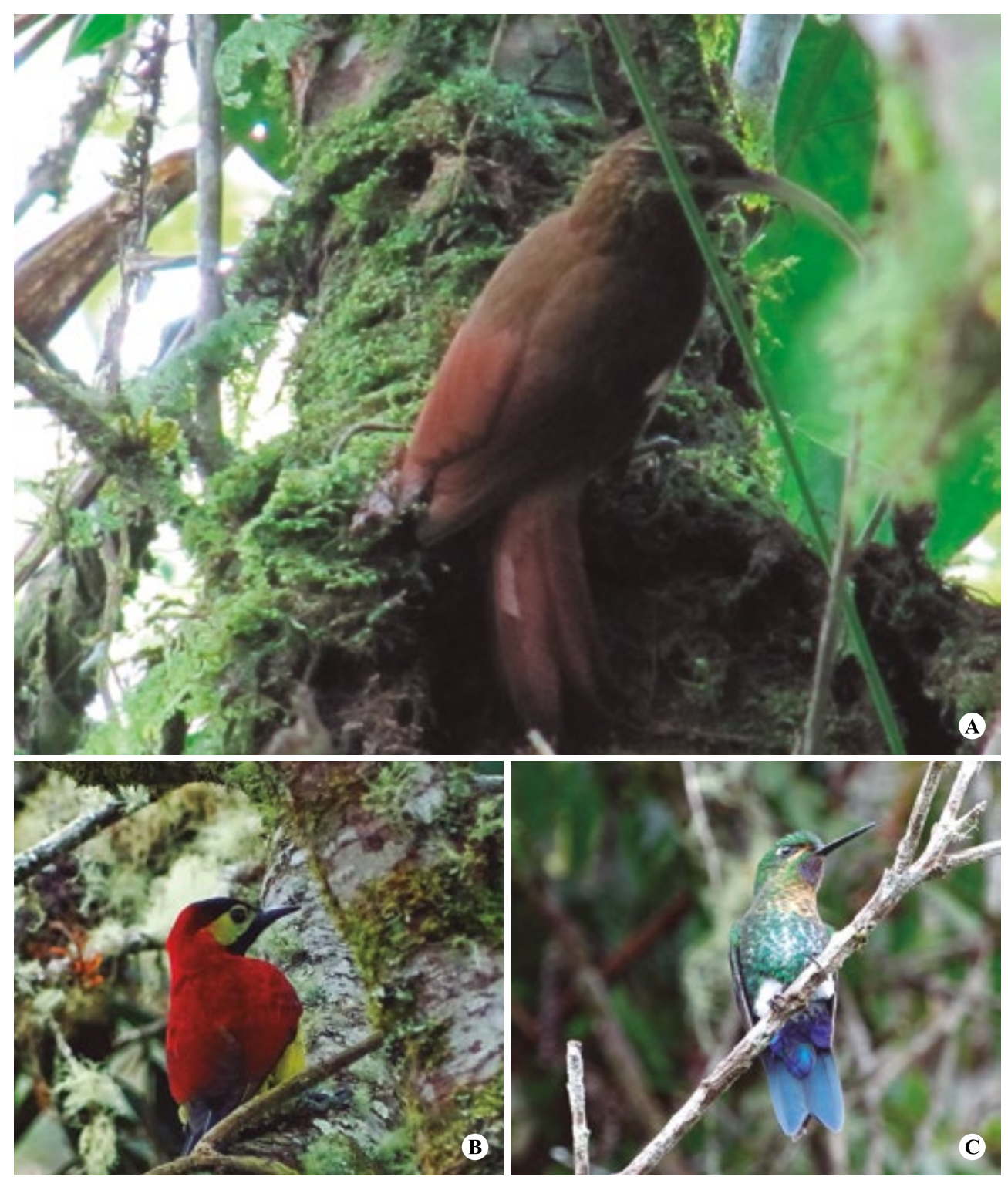

Figura 2. Tres especies con registros únicos en el PNN Farallones de Cali, donde existen los mayores vacíos de información para el municipio de Santiago de Cali. A) Drymotoxeres pucheranii (des Murs, 1849). B) Colaptes rivolii (Boissonneau, 1840). C) Eriocnemis vestita (Lesson, 1838). Fotos: A-Juan Ortíz; B y C- Danny Leandro Mora.

aves acuáticas, redescubrir especies consideradas como posiblemente extinguidas (SX) para el departamento del Valle del Cauca, confirmar registros tales como Tigrisoma fasciatum y Egretta tricolor, y evaluar el estado de conservación de especies como Porzana flaviventer.

Se sugiere también continuar colectando especímenes de aves para mejorar la representatividad de las dos colecciones del municipio que apenas alcanzan el $56,9 \%$ de representatividad (Colección Ornitológica de la Universidad del Valle y Colección Zoológica de referencia "IMCN" del Museo de Ciencias Naturales "Federico Carlos Lehmann V."). La recolección de especímenes debe estar dentro del marco de programas de investigación a largo plazo que permitan tener 
una base sólida de estudio sobre las poblaciones locales para análisis regionales (Cuervo et al. 2006), además de poder proponer y ejecutar planes específicos de conservación. En este proceso se debe rectificar y ratificar identificaciones y localidades asignadas, teniendo en cuenta las nuevas tecnologías de georreferenciación y guías especializadas de identificación.

\section{Estado de conservación de la avifauna}

En Santiago de Cali se encuentra un preocupante número de especies amenazadas, con 22 especies bajo algún grado de amenaza nacional y 86 a nivel regional. La situación podría ser más crítica debido a que el riesgo de extinción regional está basado en estudios de hace diez años (Castillo-Crespo y González-Anaya 2007), por lo cual sugerimos realizar una nueva evaluación. Para el presente estudio se identificaron de manera preliminar nueve especies más que podrían estar bajo algún grado de amenaza. En la actualidad, las principales amenazas para las aves de Santiago de Cali son la minería en las partes altas, la fragmentación y urbanización en los bosques de montaña (con pérdida de especies ya documentada; Kattan et al. 1994) y la transformación y desaparición de hábitats clave como los humedales y las madreviejas del río Cauca. En cuanto a grupos taxonómicos, los datos sugieren que las aves acuáticas y las rapaces tienen un mayor riesgo de extinción.

Las extinciones y el grado de amenaza de las especies en el municipio de Santiago de Cali son producto de procesos regionales en todo el VGRC. La transformación y desecación de los humedales, junto con la cacería que inició a mediados del siglo pasado ha llevado a la posible extirpación de cinco especies acuáticas como Netta erythrophthalma, y muchas otras tienen poblaciones en disminución como Anas cyanoptera, A. discors y Nomonyx dominicus, o ya solo quedan poblaciones domésticas como Cairina moschata y Sarkidiornis melanotos. También se reportan extirpaciones de especies como Ramphastos ambiguus, Psarocolius decumanus o Cathartes burrovianus (Lehman 1957; del Hoyo et al. 2017).
La deforestación ha permitido la colonización de varias especies desde mediados del siglo XX como Sicalis flaveola y Bubulcus ibis (Lehman 1957), y se ha incrementado a partir de la década de 1990 con apariciones de especies como Gampsonyx swainsonii (Álvarez-López y Kattan 1995), Eupsittula pertinax, Gymnomystax mexicanus (Cárdenas 1998) y Melanerpes rubricapillus (Garcés-Restrepo et al. 2012). Las consecuencias de estas colonizaciones son inciertas. También es preocupante la introducción de Lonchura malacca que puede llegar a ser una especie invasora con perjuicios económicos en la producción de gramíneas (Certuche-Cubillos et al. 2010).

La pérdida de hábitat en el bosque seco ha ocasionado la disminución de las poblaciones de varias especies, o que están siendo reemplazadas por especies de áreas abiertas. Son ejemplos el caso de Claravis pretiosa que antes era la tórtola más abundante (Lehman 1957), donde ahora domina Columbina talpacoti; la mirla Turdus obsoletus que requiere bosque y está siendo reemplazada por Turdus ignobilis la cual es oportunista y aprovecha ambientes urbanos (Vidal-Astudillo 2007); el periquito Forpus conspicillatus con poblaciones en disminución, mientras aumenta Brotogeris jugularis y el carpintero Colaptes punctigula que puede estar siendo desplazado por Melanerpes rubricapillus (GarcésRestrepo et al. 2012). En la parte plana del municipio los pastizales prácticamente han desaparecido y ha llevado a la reducción de especies como Emberizoides herbicola y la posible extirpación o declive poblacional severo de Ammodramus savannarum caucae.

\section{Recomendaciones}

La alta riqueza de especies en Santiago de Cali y su liderazgo en el suroccidente colombiano, representa una situación de responsabilidad respecto a la investigación y conservación de la avifauna a nivel regional. De hecho, el municipio se encuentra en un punto estratégico de importancia ornitológica sobre los Andes occidentales, donde se registran la mayor cantidad de especies de aves endémicas y de rangos restringidos en Colombia, las cuales son más vulnerables a la extinción (Ocampo-Peñuela y Pimm 2014). 
Resulta clave entonces el desarrollo de un modelo de conservación urbano-regional entre Santiago de Cali y municipios aledaños, especialmente Buenaventura, Dagua, Jamundí, La Cumbre y Yumbo, con el fin de promover la conservación de las aves y la biodiversidad en general, mitigar amenazas como la urbanización sin planificación y contribuir a la sustentabilidad ambiental (Andrade-Medina y Bermúdez-Cárdenas 2010, GómezContreras 2014). Se considera que la protección de los humedales remanentes es de una importancia crítica, y la articulación de propuestas para la conservación de bosques nublados desde el PNN Farallones de Cali hasta el cerro Dapa-Carisucio es clave para mejorar la conectividad entre fragmentos de bosque, la protección de la fauna y flora y garantizar el abastecimiento de servicios ecosistémicos como el agua, regulación del clima y el disfrute de la naturaleza.

\section{Agradecimientos}

Agradecemos a todas las personas e instituciones que han contribuido al conocimiento de las aves de Santiago de Cali mediante registros, listados e investigaciones, los cuales hicieron posible la elaboración de este documento.

\section{Bibliografía}

Álvarez-López, H y M. D. Heredia. 1989. Lista preliminar de las aves de Peñas Blancas. Sociedad Vallecaucana de Ornitología. Santiago de Cali, 2 pp.

Álvarez-López, H y G. H. Kattan. 1995. Notes on the conservation status of resident diurnal raptors of the middle Cauca Valley, Colombia. Bird Conservation International 5: 341-348.

Andrade-L., M. E y H. Benítez-Castañeda. 2002. Los Humedales de la Sabana de Bogotá: Área Importante para la conservación de las Aves de Colombia y el Mundo. Informe Técnico. Instituto de Investigación de Recursos Biológicos Alexander von HumboldtIAvH y BirdLife International. Bogotá D.C., 269 pp.

Andrade-Medina, P y D. C. Bermúdez-Cárdenas. 2010. La sostenibilidad ambiental urbana en Colombia. Bitácora 17 (2): 73-93.

Angarita-Martínez, I. 2002. Composición y estructura de la avifauna de la ciudad de Cali. Trabajo de grado. Universidad del Valle, Facultad de Ciencias Naturales y Exactas, Departamento de Biología. Santiago de Cali, 48 pp.

Arbeláez-Cortés, E., J. I. Garzón-Z., M. Socorro-Sierra., F. Forero., P. J. Cardona-Camacho., A. Bayer., Y. A. Beltrán-Arcila., L. M. Ramírez-Urrea., G. Patiño., J. E. Morales-Sánchez., D. DuqueMontoya y O. H. Marín-Gómez. 2015. Fourteen new additions to the list of birds of Quindío department, Colombia. Check List 11 (6): 1-9.

Arbeláez-Cortés, E., O. H. Marín-Gómez., D. Duque-Montoya., P.J. Cardona-Camacho., L.M. Renjifo y H.F. Gómez. 2011. Birds, Quindío Department, Central Andes of Colombia. Check List 7 (3): 227-247.

Asociación para el Estudio y Conservación de las Aves Acuáticas en Colombia-CALIDRIS. 2015. Censo Urbano de Aves de Cali. Asociación para el Estudio y Conservación de las Aves Acuáticas en Colombia-CALIDRIS y Departamento Administrativo de Gestión del Medio Ambiente-DAGMA. Lista de aves. Santiago de Cali, Colombia.

Asociación para el Estudio y Conservación de las Aves Acuáticas en Colombia-CALIDRIS. 2016. Censo Urbano de Aves de Cali. Asociación para el Estudio y Conservación de las Aves Acuáticas en Colombia-CALIDRIS y Departamento Administrativo de Gestión del Medio Ambiente-DAGMA. Lista de aves. Santiago de Cali, Colombia.

Ayerbe-Quiñones, F., L. G. Gómez-Bernal, J. P. López-Ordóñez, M. Ramírez-Burbano, J. V. Sandoval-Sierra y M. F. González-Rojas. 2009. Avifauna de Popayán y municipios aledaños. Novedades Colombianas 9(1): 1-27.

Bacon J., E. Velasco y A. Lieberman. 1985. Aves de El Topacio, Parque Nacional Natural Farallones de Cali, Frente Cali. En: Sarria-Salas, S. 1993. Parque Nacional Natural Farallones de Cali. Monografía. Corporación Autónoma Regional del Valle del Cauca-CVC y Fundación Protectora de las CuencasPROCUENCAS. Santiago de Cali, 313 pp.

Barve, N., V. Barve., A. Jiménez-Valverde, A. Lira-Noriega, S. P. Maher, A. T. Peterson, J. Soberón y F. Villalobos. 2011. The crucial role of the accessible area in ecological niche modeling and species distribution modeling. Ecological Modelling 222: 1810-1819.

BirdLife International. 2017. Important Bird Areas factsheet: Bosque de San Antonio/Km 18. Descargado de: $<$ http://datazone. birdlife.org/site/factsheet/bosque-de-san-antonio-km-18-ibacolombia> on $18 / 02 / 2017$

Botero, J. E., G. M. Lentijo, A. M. López, O. Castellanos, C. Aristizabal., N. Franco y D. Arbeláez. 2005. Adiciones a la lista de aves del municipio de Manizales. Boletín SAO 15 (2): 69-88.

Calvert, L. 2016. Lista de aves reportadas en la finca La Esmeraldacorregimiento de Pichindé, Santiago de Cali, Valle del Cauca.

Cantera-K., J. R. 2010. Vida silvestre en el campus de la Universidad del Valle. Programa Editorial Universidad del Valle. Facultad de Ciencias Naturales y Exactas. Departamento de Biología. Santiago de Cali, 275 pp.

Cárdenas, G. 1998. Comparación de la composición y estructura de la avifauna en diferentes sistemas de producción. Trabajo de grado. Universidad del Valle, Facultad de Ciencias Naturales y Exactas, Departamento de Biología. Santiago de Cali, 68 pp.

Cárdenas, G y V. Vidal-Astudillo. 2010. Lista de Aves de los Ecoparques Urbanos Bataclán y Písamos. Informe Preliminar. Departamento Administrativo de Gestión del Medio AmbienteDAGMA. Santiago de Cali, 9 pp.

Castillo-Crespo, L. S y M. González-Anaya. (Eds.-Comp.). 2007. Avances en la implementación del plan de acción en biodiversidad del Valle del Cauca. Corporación Autónoma 
Regional del Valle del Cauca-CVC, Dirección Técnica Ambiental, Grupo Biodiversidad. Santiago de Cali, 66 pp.

Certuche-Cubillos, K., D. Carantón-Ayala., R. M. ParraHernández., M. Moreno-Palacios., C. Díaz-Jaramillo y J. Sanabria-Mejía. 2010. Biología alimentaria del Capuchino de cabeza negra (Lonchura malacca, Estrildidae) en el alto valle del Magdalena, Colombia. Ornitología Colombiana 9: 25-30.

Chaparro-Herrera S., M. A Echeverry-Galvis, S. Córdoba-Córdoba y A. Sua-Becerra. 2013. Listado actualizado de las aves endémicas y casi-endémicas de Colombia. Biota Colombiana 14 (2): 235-272.

Chapman, F. M. 1917. The distribution of bird-life in Colombia: a contribution to a biological survey of the South America. Bulletin of the American Museum of Natural History Volume XXXVI. New York, USA. 836 pp.

CIAT-Centro Internacional de Agricultura Tropical, Corporación Autónoma Regional del Valle del Cauca-CVC y Departamento Administrativo de Gestión del medio Ambiente-DAGMA. 2016. Estudio para la microzonificación climática para el municipio de Santiago de Cali. Centro Internacional de Agricultura TropicalCIAT. Santiago de Cali, 44 pp.

Cifuentes-Sarmiento, Y. y C. Ruiz-Guerra. 2016. Aves de la finca Santa Helena, Estación MoSI (Monitoreo de Sobrevivencia Invernal) en Colombia. Informe Final. Asociación para el Estudio y Conservación de las Aves Acuáticas en Colombia-CALIDRIS. Santiago de Cali, Colombia. 11 pp.

Consorcio C.S.C.-2009. 2011. Estudio de factibilidad técnica, financiera, económica, social y ambiental para construcción de un embalse de regulación sobre el río Cali y protección integral de su cuenca a través de la gestión integral del recurso. Etapa II: Diseño Básico del Proyecto. Informe Línea Base Fauna y Flora. Documento No. I-T0379- DPCL-3900-007. Santiago de Cali, 55 pp.

Contreras-Rengifo, R. 2006. Formulación del plan de manejo integral del humedal El Estero, en el corregimiento de Navarro, municipio de Cali, Valle del Cauca. Corporación Autónoma Regional del Valle del Cauca-CVC. Santiago de Cali, 75 pp.

Contreras-Rengifo, R., R. Ríos-Herrera, M. C. Serrato, I. Cabrera y R. Domínguez. 2006. Formulación del plan de manejo integral del humedal Marañón en el corregimiento El Hormiguero, municipio de Cali, Valle del Cauca. Informe Final. Santiago de Cali, Colombia. 118 pp.

Corporación Autónoma Regional del Valle del Cauca-CVC y Fundación Mundo Ambiental. 2006. Plan de manejo ambiental humedal Navarro, municipio de Santiago de Cali. Corporación Autónoma Regional del Valle del Cauca-CVC. Dirección Técnica Ambiental. Santiago de Cali, 147 pp.

Cuervo, A. M., C. D. Cadena y J. L. Parra. 2006. Seguir colectando aves en Colombia es imprescindible: Un llamado a fortalecer las colecciones ornitológicas. Ornitología Colombiana 4: 51-58.

DAGMA-Departamento Administrativo de Gestión del Medio Ambiente. 2010. Las aves de mi ciudad: Una guía de las aves de Cali. Departamento Administrativo de Gestión del Medio Ambiente-DAGMA y Asociación para el Estudio y la Conservación de las Aves Acuáticas en Colombia-Calidris. Santiago de Cali, 123 pp.

DAPM-Departamento Administrativo de Planeación Municipal. 1996. Estudio ecológico ambiental de la Reserva Forestal del municipio de Santiago de Cali, Fase I. Informe sectoriales de fauna y flora. Ingenieros Consultores S.A.-INGECON S.A. y Municipio de Santiago de Cali. Santiago de Cali, Colombia.

DAPM-Departamento Administrativo de Planeación Municipal. 2016. Cali en cifras 2015. Departamento Administrativo de Planeación Municipal-DAPM. Santiago de Cali, 211 pp.

del Hoyo, J., Elliott, A., Sargatal, J., Christie, D. A y E. de Juana. (Eds.) 2017. Handbook of the birds of the world alive. Lynx Editions, Barcelona. (Descargado de $<\mathrm{http}$ :/www.hbw.com/> on 24/03/2017).

Delgado-V, C. A y J. C. Correa-H. 2013. Estudios ornitológicos urbanos en Colombia: revisión de literatura. Revista Ingeniería y Ciencia 19: 215-236.

Downer, C. C. 1978. Lista de las aves en el Parque Nacional Natural de los Farallones con descripciones y distribuciones de los órdenes, familias y especies. En: Sarria-Salas, S. 1993. Parque Nacional Natural Farallones de Cali. Monografía. Corporación Autónoma Regional del Valle del Cauca-CVC y Fundación Protectora de las Cuencas-PROCUENCAS. Santiago de Cali, $313 \mathrm{pp}$.

Downer, C. C. 1979a. Lista de anfibios, mamíferos y aves encontradas en los Altos Farallones. En: Sarria-Salas, S. 1993. Parque Nacional Natural Farallones de Cali. Monografía. Corporación Autónoma Regional del Valle del Cauca-CVC y Fundación Protectora de las Cuencas-PROCUENCAS. Santiago de Cali, 313 pp.

Downer, C. C. 1979b. Mamíferos y aves de la zona alta del Parque Nacional Natural Farallones de Cali. En: Sarria-Salas, S. 1993. Parque Nacional Natural Farallones de Cali. Monografía. Corporación Autónoma Regional del Valle del Cauca-CVC y Fundación Protectora de las Cuencas-PROCUENCAS. Santiago de Cali, 313 pp.

eBird Basic Dataset. Version: EBD_relNov-2016. Cornell Lab of Ornithology, Ithaca, New York. Nov 2016.

Estela, F. A., D. Arbeláez., D. Fajardo., L. A. Neira y S. Restrepo. 2004. Caracterización ornitológica del páramo del Duende y su zona de amortiguación. Informe Técnico. Asociación para el Estudio y la Conservación de las Aves Acuáticas en ColombiaCalidris. Santiago de Cali, 14 pp.

Estrada, M. C. 2014. La biodiversidad como soporte para la toma de decisiones en el territorio de la ciudad de Medellín: Aves. Pp: 48-55. En: Alcaldía de Medellín (Ed.). Propuesta para la gestión integral de la biodiversidad y los servicios ecosistémicos en Medellín: Síntesis del documento técnico de soporte. Parque Explora. Medellín, 240 pp.

Fuentes-Pardo, G. E. 2010. Caracterización de la avifauna del Jardín Botánico de Cali y Ecoparque Río Cali. Trabajo de grado. Universidad del Valle, Facultad de Ciencias Naturales y Exactas, Departamento de Biología. Santiago de Cali, 67 pp.

Garcés-Restrepo, M. F., C. A. Saavedra-Rodríguez, G. Cárdenas, V. Vidal-Astudillo, F. Ayerbe-Quiñones, L. F. Ortega, J. E. López-Solarte, R. Johnston-González y C. A. Ríos-Franco. 2012. Expansión de la distribución y datos ecológicos del Carpintero Habado (Melanerpes rubricapillus) en el valle del río Cauca, Colombia. Ornitología Colombiana 12: 54-60.

Gniadek, S. 1973. Las aves de "La Margarita", Peñas Blancas, río Pichindé, Cali, Valle del Cauca. Cuerpo de Paz/Corporación Autónoma Regional del Valle del Cauca-CVC. En: Sarria-Salas, 
S. 1993. Parque Nacional Natural Farallones de Cali. Monografía. Corporación Autónoma Regional del Valle del Cauca-CVC y Fundación Protectora de las Cuencas-PROCUENCAS. Santiago de Cali, 313 pp.

Gómez-Contreras, J. L. 2014. Del desarrollo sostenible a la sustentabilidad ambiental. Revista Facultad de Ciencias Económicas 14 (1): 115-136.

Hernández-C, O., V. Cardona-B y P. Montoya-V. 2015. Riqueza de especies de aves en el campus de la Universidad del Valle, once años después. Revista Colombiana de Ciencia Animal-RECIA 7 (1): 25-34.

IUCN. 2016. The IUCN red list of threatened species. Version 2016-3.

Jordán-Muñoz, J. F., J. A. Restrepo, J. Luna-Solarte, P. A. LunaSolarte, A. Muñoz y T. Muñoz. (Sin año). Listados de aves del corregimiento La Leonera, municipio de Santiago de Cali. Lista de aves. Cali, Colombia. En: Vidal-Astudillo, V. 2010. Recopilación de las especies de vertebrados reportados en las cuencas de los ríos Felidia y Pichindé, Municipio de Santiago de Cali. Informe técnico. Consorcio C.S.C. 2009. Santiago de Cali, 49 pp.

Kattan, G., H. Álvarez-López y M. Giraldo. 1991. Efectos de la fragmentación de bosques sobre la composición de la avifauna: San Antonio 80 años después. Informe de Investigación, Proyecto No. 366. Fundación para la Promoción de la Investigación y la Tecnología-FPIT. Instituto Vallecaucano de Investigaciones Científicas y Departamento de Biología, Universidad del Valle. Banco de la República. Santiago de Cali, Colombia.

Kattan, G. H., H. Álvarez-López y M. Giraldo. 1994. Forest fragmentation and bird extinctions: San Antonio eighty years later. Conservation Biology 8 (1): 138-146.

Kattan, G. H., S. A. Tello., M. Giraldo y C. D. Cadena. 2016. Neotropical bird evolution and 100 years of the enduring ideas of Frank M. Chapman. Biological Journal of the Linnean Society 117 (3): 407-413.

Lehmann, F. C. 1957. Contribuciones al estudio de la fauna de Colombia XII. Novedades Colombianas 1: 101-156.

Londoño-Betancourth, J. C. 2011. Una mirada a la diversidad ornitológica de Pereira. Boletín Científico Museo de Historia Natural 15: 84-103.

López-Carvajal, H. 2012. Fauna terrestre río Meléndez zona del Club Campestre Cali. Proyecto Urbanístico CUSEZAR. Informe Final. Santiago de Cali, 10 pp.

López-Carvajal, H. 2014. Fauna terrestre del humedal de la Universidad Javeriana Cali. Informe Final. Santiago de Cali, $21 \mathrm{pp}$.

López-Carvajal, H. 2016. Avifauna del Cerro Los Cristales. Lista de Aves. Santiago de Cali, 2 pp.

Miller, A. H. 1963. Seasonal activity and ecology of the avifauna of an American equatorial cloud forest. University of California Publications in Zoology 66 (1): 106-124.

Montealegre-Talero, C. 2009. Patrones de diversidad en ensamblajes de aves en dos bosques de niebla de la Cordillera Occidental de Colombia. Trabajo de grado. Universidad de los Andes, Facultad de Ciencias, Departamento de Ciencias Biológicas. Bogotá, D.C. 17 pp.
Muñoz M. C., K. Fierro-Calderón y H. F. Rivera-Gutiérrez. 2007. Las aves del campus de la Universidad del Valle, una isla verde urbana en Cali, Colombia. Ornitología Colombiana 5: 5-20.

Muñoz-A., L. A. y A. M. Carmona-T. (Sin año a). Plan de manejo ambiental humedal urbano Lago de Las Garzas. Departamento Administrativo de Gestión del Medio Ambiente-DAGMA y Subdirección de Gestión Ambiental-SGA. Santiago de Cali, Colombia. 227 pp.

Muñoz-A., L. A. y A. M. Carmona-T. (Sin año b). Plan de manejo ambiental humedal urbano Lago de Los Cisnes. Departamento Administrativo de Gestión del Medio Ambiente-DAGMA y Subdirección de Gestión Ambiental-SGA. Santiago de Cali, Colombia. 242 pp.

Muñoz-A., L. A. y A. M. Carmona-T. (Sin año c). Plan de Manejo Ambiental Humedal Urbano Lago Panamericano. Departamento Administrativo de Gestión del Medio Ambiente-DAGMA y Subdirección de Gestión Ambiental-SGA. Santiago de Cali, $219 \mathrm{pp}$.

Naranjo, L. G. y F. Estela. 1999. Inventario de la avifauna de un área suburbana de la ciudad de Cali. Boletín SAO. 10 (18-19): 11-27.

Naranjo, L. G., J.D. Amaya., D. Eusse-González y Y. CifuentesSarmiento (Eds.). 2012. Guía de las especies migratorias de la biodiversidad en Colombia. Aves. Vol. 1. Ministerio de Ambiente y Desarrollo Sostenible-MADS y Fondo Mundial para la Naturaleza-WWF Colombia. Bogotá D.C., 708 p.

Ocampo-Peñuela, N. y S. L. Pimm. 2014. Setting practical conservation priorities for birds in the western Andes of Colombia. Conservation Biology 28(5): 1260-1270.

Ortega-Álvarez, R. y I. MacGregor-Fors. 2011. Dusting-off the file: A review of knowledge on urban ornithology in Latin America. Landscape and Urban Planning 101 (1): 1-10.

Orejuela-Gartner, J. E., N. Correa-Ortiz, H. López-Carvajal, V. Vidal-Astudillo y C. M. Wagner-Wagner. 2007. Evaluar el estado de la fauna y flora del bosque de San Antonio, área de interés ambiental de la cuenca del río Cali, municipio de Cali. Informe Final. Convenio CVC 051 de 2007. Corporación Autónoma Regional del Valle del Cauca-CVC, Dirección Técnica Ambiental, Grupo Biodiversidad y Asociación Río Cali-ASORÍOCALI. Santiago de Cali, 96 pp.

Parra-Hernández, R. M., D. A. Carantón-Ayala, J. S. SanabriaMejía, L. F. Barrera-Rodríguez, A. M. Sierra-Sierra, M. C. Moreno-Palacios, W. S. Yate-Molina, W. E. Figueroa-Martínez, C. Díaz-Jaramillo, V. T. Florez-Delgado, J. K. Certuche-Cubillos, H. N. Loaiza-Hernández y B. A. Florido-Cuellar. 2007. Aves del municipio de Ibagué-Tolima, Colombia. Biota Colombiana 8(2): 199-220.

Ramírez-Lamus, J. C., G. L. Vásquez-Zapata., A. M. NavarreteGálvez y J. E. Orejuela Gartner. 2001. Determinación del estado sucesional de los humedales: madrevieja Guarinó, ciénaga La Guinea, caño El Estero, laguna Pacheco, madrevieja Lili, madrevieja Román (Gota e'leche), madrevieja Chiquique, madrevieja La Herradura y laguna Bocas del Tuluá, localizadas en los municipios de Cali, Jamundí, Bolívar y Tuluá, departamento del Valle del Cauca. Informe Final. Contrato CVCSGA-069-2000. Corporación Autónoma Regional del Valle del Cauca-CVC y SGA. Santiago de Cali, 264 pp.

Remsen, J. V. Jr. Areta, J. I. Cadena, C. D. Claramunt, S. Jaramillo, A. Pacheco, J. F. Pérez-Emán, J. Robbins, M. B. Stiles, F. 
G. Stotz, D. F. and K. J. Zimmer. Version [10/03/2017]. A classification of the bird species of South America. American Ornithologists' Union.

Renjifo, L. M., M. F. Gómez, J. Velásquez-Tibatá, J., A. M. Amaya-Villarreal., G. H. Kattan., J.D. Amaya-Espinel y J. Burbano-Girón. 2014. Libro rojo de aves de Colombia, Volumen I: Bosques Húmedos de los Andes y la costa Pacífica. Editorial Pontificia Universidad Javeriana e Instituto Alexander von Humboldt. Bogotá D.C., 466 pp.

Renjifo, L. M., A. M. Amaya-Villarreal., J. Burbano-Girón y J. Velásquez-Tibatá. 2016. Libro rojo de aves de Colombia, Volumen II: Ecosistemas abiertos, secos, insulares, acuáticos continentales, marinos, tierras altas del Darién y Sierra Nevada de Santa Marta y bosques húmedos del centro, norte y oriente del país. Editorial Pontificia Universidad Javeriana e Instituto Alexander von Humboldt. Bogotá D.C., 563 pp.

Reyes-Gutiérrez, M. y S. Restrepo-Calle. 2005. Las aves del Ecoparque Río Pance. Corporación Autónoma Regional del Valle del Cauca-CVC, Corporación para la Recreación Popular y Fondo para la Acción Ambiental. Santiago de Cali, 72 pp.

Reyes-Gutiérrez, M., R. Sedano y S. M. Durán. 2002. Lista anotada de la avifauna de la Universidad del Valle, Cali, Colombia. Boletín SAO 13 (24-25):12-25.

Riascos-Vallejo, J. M. 2001. Aves, mamíferos, reptiles y anfibios de la Colección Zoológica de referencia "IMCN" del Museo de Ciencias Naturales "Federico Carlos Lehmann V." Cespedesia 24 (75-78): 95-152.

Rivera-Gutiérrez, H. F. 2006. Composición y estructura de una comunidad de aves en un área suburbana en el suroccidente Colombiano. Ornitología Colombiana 4: 28-38.

Salazar-Mancipe, A. M., C. M. Vásquez-Camargo y G. L. VásquezZapata. 2006. Plan de manejo integral humedal Cañasgordas, municipio de Santiago de Cali. Contrato de Consultoría No. 557-06. DAGMA y Fundación Río Cauca. Departamento Administrativo de Gestión del Medio Ambiente-DAGMA y Fundación Río Cauca. Santiago de Cali, Colombia. 147 pp.

Sarria-Salas, S. 1993. Parque Nacional Natural Farallones de Cali. Monografía. Corporación Autónoma Regional del Valle del Cauca-CVC y Fundación Protectora de las CuencasPROCUENCAS. Santiago de Cali, 313 pp.

Sullivan, B. L., C. L. Wood, M. J. Iliff, R. E. Bonney, D. Fink y S. Kelling. 2009. eBird: a citizen-based bird observation network in the biological sciences. Biological Conservation 142: 2282-2292.
UAESPNN-Unidad Administrativa Especial del Sistema de Parques Nacionales Naturales. 2005. Plan de Manejo 20052009. Parque Nacional Natural Farallones de Cali. Unidad Administrativa Especial del Sistema de Parques Nacionales Naturales-UAESPNN. Santiago de Cali, 338 pp.

Universidad del Valle. 2014. Colección Ornitológica de la Universidad del Valle, 5361 registros, aportados por CruzBernate, L. (Curador de la Colección, Contacto del recurso, Proveedor de los Metadatos), Tamayo-Quintero, J. (Creador del recurso), <http://ipt.sibcolombia.net/valle/resource. do? $\mathrm{r}=$ coleccion_ornitologica_universidad_del_valle $>$, versión 5 (actualizado el 25/11/2014).

Vargas, F., Muñoz, N. J., Londoño, J. E. y R. Pardo. 2007. Elaborar cinco estudios de capacidad de carga en la zona rural del municipio de Santiago de Cali. Informe Final. Contrato No. 023 de 2007. Unidad Municipal de Asistencia Técnica AgropecuariaUMATA y Fundación para la Vida en Comunidad-FUNVIVIR. Santiago de Cali, 158 pp.

Verhelst, J. C., Rodríguez, J. C., Orrego, O., Botero, J. E., López, J. A., Franco, V. M. y A. M. Pfeifer. 2001. Aves del Municipio de Manizales- Caldas, Colombia. Biota Colombiana 2 (3): 265-284.

Vidal-Astudillo, V. 2010. Recopilación de las especies de vertebrados reportados en las cuencas de los ríos Felidia y Pichindé, Municipio de Santiago de Cali. Informe Técnico. Consorcio C.S.C. 2009. Santiago de Cali, 49 pp.

Vidal-Astudillo, V. 2013. Lista de chequeo de las aves del Club Campestre de Cali y sus alrededores. Club Campestre de Cali, Asociación Río Cali-ARC y Mapalina-Birding Trails. Imprenta Imágenes Gráficas. Santiago de Cali, 52 pp.

Vidal-Astudillo, V., Cárdenas, G., Ortega-G., L. F., SaavedraRodríguez, C. A. y M. F. Garcés-Restrepo. 2008. Monitoreo permanente sobre el estado y dinámica de las poblaciones de avifauna para medir el impacto generado por la intervención y compensación forestal de este ecosistema urbano de La Calle Quinta. Informe Final. CONALVIAS S.A. Santiago de Cali, $82 \mathrm{pp}$.

Vidal-Astudillo, V. 2007. Observaciones del comportamiento de forrajeo y reproductivo de la Mirla ollera Turdus ignobilis (Aves: Turdidae) relacionado con la precipitación en el corregimiento Villa Carmelo-Valle del Cauca. Trabajo de grado. Universidad del Valle, Facultad de Ciencias Naturales y Exactas, Departamento de Biología. Santiago de Cali, 39 pp.

Vidal-Astudillo, V. y C. M. Wagner. 2010. Listado de especies de aves registradas en el predio Bichacue Yath, corregimiento La Leonera. Informe Final. Santiago de Cali, 2 pp. 
Rubén Darío Palacio

Fundación Ecotonos

Santiago de Cali, Valle del Cauca, Colombia

Nicholas School of the Environment, Duke University, Durham, Estados Unidos

rdpalacio@ecotonos.org

\section{Viviana Vidal-Astudillo}

Vidal \& Astudillo Ltda.

Asociación para el Estudio y la Conservación de las Aves Acuáticas en Colombia -Calidris

Santiago de Cali, Valle del Cauca, Colombia

viviana@vidalastudillo.com

\section{Giovanni Cárdenas}

Asociación para el Estudio y la Conservación de las Aves Acuáticas en Colombia -Calidris

Santiago de Cali, Valle del Cauca, Colombia

gcardenas@calidris.org.co

José Luna-Solarte

Colombia Birdwatch

joselunasolarte@gmail.com
Aves del municipio de Santiago de Cali, Valle del Cauca, Colombia

Citación del artículo: Palacio, R. D., V. Vidal-Astudillo, G. Cárdenas y J. Luna-Solarte. 2017. Aves del municipio de Santiago de Cali, Valle del Cauca, Colombia. Biota Colombiana 18 (2): 228-238. DOI: 10.21068/c2017.v18n02a15

Recibido: 12 de junio de 2017

Aprobado: 25 de julio de 2017 